\title{
A multidrug-resistant Stenotrophomonas maltophilia clinical isolate from Kamuzu Central Hospital, Malawi
}

\section{Geoffrey Peterkins Kumwenda ${ }^{1}$, Watipaso Kasambara ${ }^{1}$, Kenneth Chizani ${ }^{1}$, Abel Phiri ${ }^{1}$, Alick Banda ${ }^{1}$, Faheema Choonara $^{2}$, Burnet Lichapa ${ }^{3}$}

1. Ministry of Health, Microbiology National Reference Laboratory, Lilongwe, Malawi

2. Ministry of Health, Kamuzu Central Hospital Laboratory, Lilongwe, Malawi

3. Dignitas International, Zomba, Malawi

Correspondence: Geoffrey P. Kumwenda (geoffreykumwenda@gmail.com)

\begin{abstract}
Background
Abstract

Stenotrophomonas maltophilia is a significant opportunistic pathogen that is associated with high mortality in immunocompromised individuals. In this study, we describe a multidrug-resistant (MDR) S. maltophilia clinical isolate from Kamuzu Central Hospital (KCH), Lilongwe, Malawi.

Methods

A ceftriaxone and meropenem nonsusceptible isolate (Sm-MW08), recovered in December 2017 at KCH, was referred to the National Microbiology Reference Laboratory for identification. In April 2018, we identified the isolate using MALDI Biotyper mass spectrometry and determined its antimicrobial susceptibility profile using microdilution methods. Sm-MW08 was analysed by S1PFGE, PCR, and Sanger sequencing, in order to ascertain the genotypes that were responsible for the isolate`s multidrug-resistance (MDR) phenotype.

Results

Sm-MW08 was identified as $S$. maltophilia and exhibited resistance to a range of antibiotics, including all $\beta$-lactams, aminoglycosides (except arbekacin), chloramphenicol, minocycline, fosfomycin and fluoroquinolones, but remained susceptible to colistin and trimethoprim-sulfamethoxazole. The isolate did not harbour any plasmid but did carry chromosomally-encoded $b{ }_{\mathrm{L} 1}$ metallo- $\beta$ lactamase and $b l a_{\mathrm{L} 2} \beta$-lactamase genes; this was consistent with the isolate's resistance profile. No other resistance determinants were detected, suggesting that the MDR phenotype exhibited by Sm-MW08 was innate.

Conclusion

Herein, we have described an MDR S. maltophilia from KCH in Malawi, that was resistant to almost all locally available antibiotics. We therefore recommend the practice of effective infection prevention measures to curtail spread of this organism.
\end{abstract}

Key Words: Stenotrophomonas maltophilia, ceftriaxone, carbapenem, Kamuzu Central Hospital, Malawi

\section{Introduction}

Reports of multidrug-resistant (MDR) bacteria have dramatically increased, posing a threat to medical interventions. One such organism is Stenotrophomonas maltophilia, a significant opportunistic pathogen that can be fatal in immunocompromised individuals. This organism is associated with mortality rates as high as $69 \%$ in systemic infections ${ }^{1}$. The World Health Organization (WHO) has listed S. maltophilia as one of the emerging pathogens that are of importance to public health ${ }^{2}$. This gram-negative bacterium is usually inherently resistant to some common antibiotics ${ }^{3}$ but can acquire additional resistance horizontally, ultimately rendering almost all antibiotics ineffective ${ }^{3}$.

In Malawi, ceftriaxone, sulfamethoxazole-trimethoprim, aminoglycosides, and fluoroquinolones, are the most commonly used antibiotics to treat gram-negative bacterial infections. In this report, we describe an MDR S. maltophilia isolate from Kamuzu Central Hospital (KCH) in Lilongwe, Malawi. We also provide an antimicrobial susceptibility profile to inform us of potential therapeutic options for treating infections caused by this isolate.

\section{Methods}

A ceftriaxone and meropenem nonsusceptible gram-negative bacillus (designated Sm-MW08) was recovered at $\mathrm{KCH}$ from a blood sample taken from a patient admitted to the medical ward high-dependency unit in December 2017. The isolate was submitted to the Malawi National Microbiology Reference Laboratory (MNMRL) for identification and further microbiological investigation. Sm-MW08 was anonymized at $\mathrm{KCH}$ laboratory prior to submission. In April 2018, we revived the isolate from a glycerol stock using brain heart infusion (BHI) agar and identified it by mass spectrometry using a MALDI Biotyper system (Bruker Daltonics GmbH, Leipzig, Germany). Profiling of the antimicrobial susceptibility pattern of Sm-MW08 was performed by determining the minimum inhibitory concentrations (MICs) of various antibiotics using the MicroScan WalkAway Plus system (Beckman Coulter, CA, USA) or Eiken dry plates (Eiken, Tokyo, Japan). Results were interpreted according to CLSI version M100-S24 or EUCAST version 9.0 guidelines.

Since Sm-MW08 initially exhibited nonsusceptibility to meropenem and ceftriaxone, we screened the isolate for carbapenemases (these confer resistance to almost all $\beta$-lactam antibiotics) using the modified carbapenem inactivation method according to the CLSI protocol. We also tested the isolate for the production of extended-spectrum $\beta$-lactamases (using cefotaxime + clavulanic acid); these enzymes confer resistance to aztreonam and oxyimino-cephalosporins (e.g., 


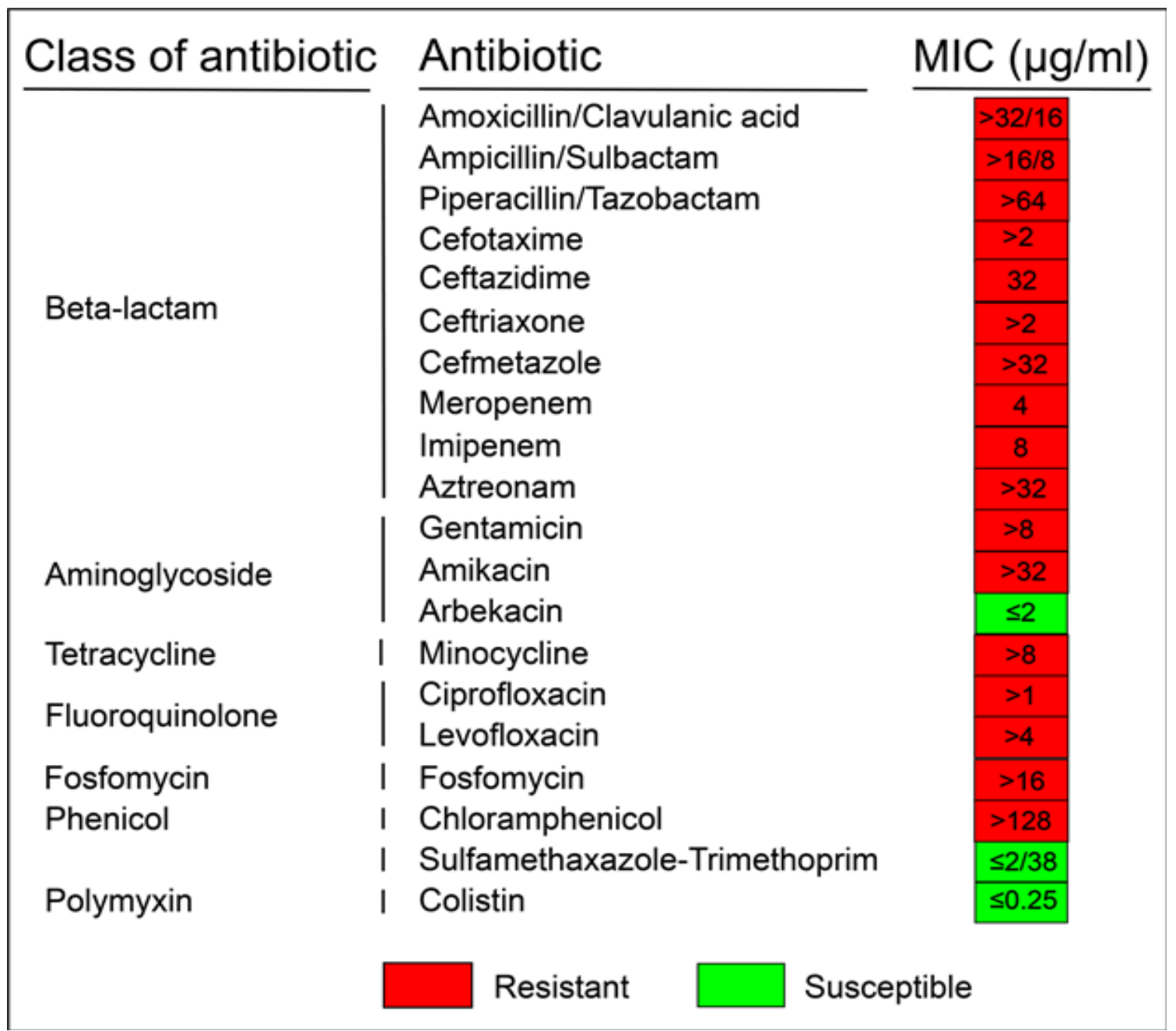

Figure 1: Antimicrobial susceptibility profile of S. maltophilia Sm-MW08 clinical isolate recovered at Kamuzu Central Hospital, Malawi. All results were interpreted using CLSI version M100-S24 guidelines except for colistin which was interpreted using EUCAST version 9.0 guidelines and arbekacin which was defined based on previous reports. MIC, minimum inhibitory concentration.

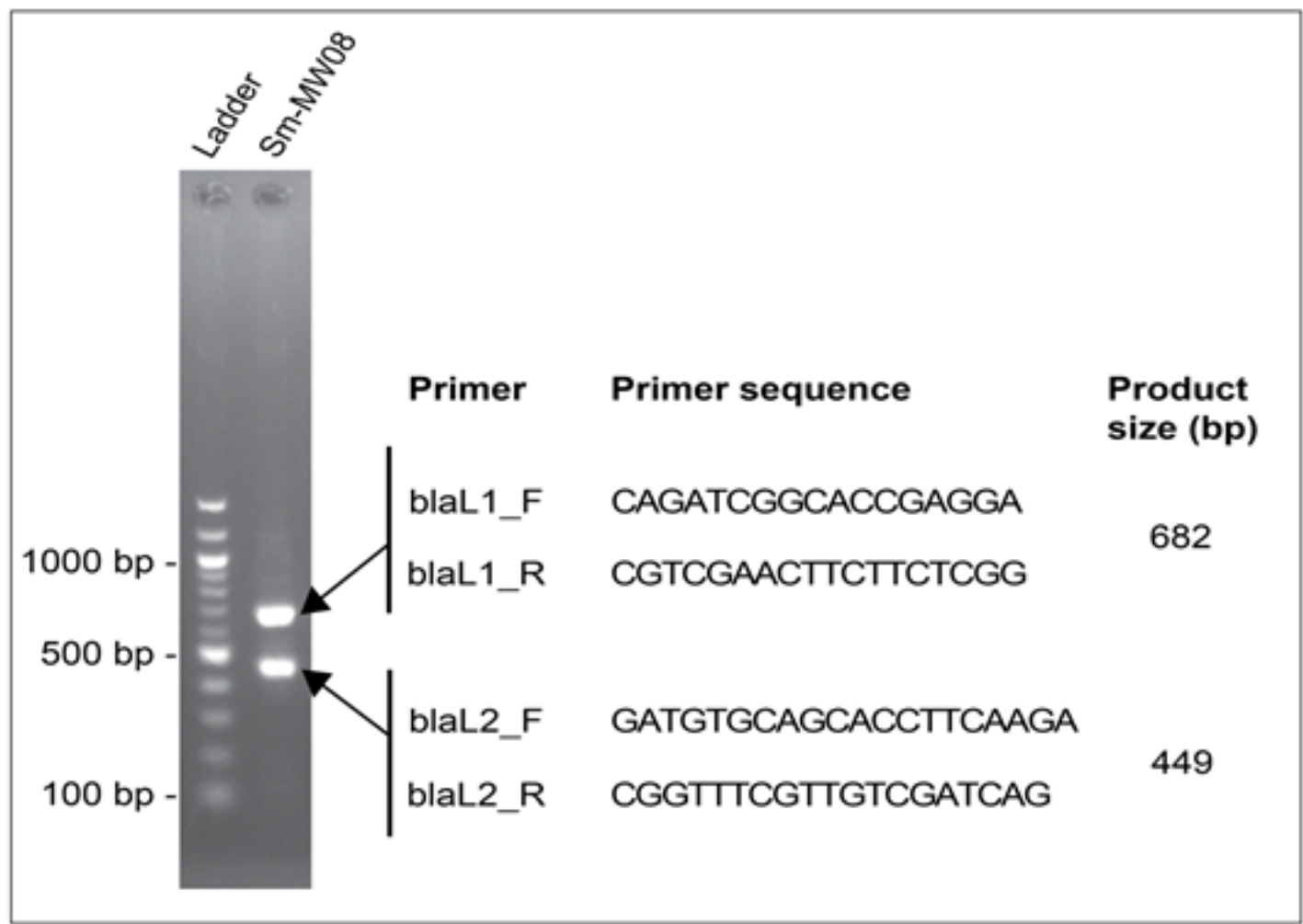

Figure 2: S. maltophilia Sm-MW08 harboured both bla metallo- $\beta$-lactamase and bla $\beta$-lactamase genes. Bacterial DNA was extracted using PowerSoil DNA isolation kit (Qiagen) followed by a multiplex PCR using Ex-Taq DNA polymerase (TaKaRa) at $98^{\circ} \mathrm{C}$ initial denaturation for $3 \mathrm{~min}, 35 \mathrm{cycles}$ of $98^{\circ} \mathrm{C}$ denaturation for $30 \mathrm{~s}, 60^{\circ} \mathrm{C}$ annealing for $30 \mathrm{~s}$ and $72^{\circ} \mathrm{C}$ extension for 40 s. PCR products were analysed by Electrophoresis on a $2 \%$ gel. Primer sequences were designed during this study. 
cefotaxime, ceftazidime and ceftriaxone). We also used PCR to screen Sm-MW08 for carbapenemase-encoding genes $\left(b l a_{\mathrm{NDM}}, b l a_{\mathrm{KPC}}, b l a_{\mathrm{OXA}-48-\mathrm{Like}}\right.$ and $\left.b l a_{\mathrm{L} 1}\right)$ and other $\beta$-lactamase genes $\left(b l a_{\text {CTX-M }}, b l a_{\text {SHY }} b l a_{\text {TEM }}\right.$, and $\left.b l a_{\mathrm{L} 2}\right)$. Next, we used PCR and Sanger sequencing to screen Sm-MW08 for mutations within the quinolone resistance determining region (QRDR); this region is known to confer quinolone/fluoroquinolone resistance. Finally, to investigate the possibility of acquired resistance, we analysed Sm-MW08 for plasmids by S1 pulsedfield gel-electrophoresis (PFGE).

\section{Results}

Sm-MW08 was identified as S. maltophilia. The isolate was resistant to almost all of the antibiotics tested, including all $\beta$-lactams (including meropenem and $\beta$-lactamase inhibitor combinations), gentamicin, amikacin, minocycline, ciprofloxacin, levofloxacin, fosfomycin, and chloramphenicol, but remained susceptible to arbekacin, sulfamethoxazoletrimethoprim, and colistin (Figure 1). PCR revealed that Sm-MW08 harboured $b l a_{\mathrm{L} 1}$ metallo- $\beta$-lactamase and $b l a_{\mathrm{L} 2}$ $\beta$-lactamase (Figure 2); these findings were confirmed by Sanger sequencing. The hydrolytic activities of L1 and L2 were confirmed by disc inactivation and double disc tests, respectively. We did not detect any plasmids in S. maltophilia Sm-MW08 or any other resistance determinants or mutations within the QRDR.

\section{Discussion}

MDR S. maltophilia is an emerging pathogen that can cause lifethreatening infections in immunocompromised individuals. S. maltophilia Sm-MW08 was recovered from a bacteraemic patient at $\mathrm{KCH}$. The isolate exhibited resistance to almost all tested antibiotics, but remained susceptible to one locally available antibiotic, sulfamethoxazole-trimethoprim; this is a recognized drug option for the treatment of $S$. maltophilia infections. ${ }^{3,4}$. However, bacteria with plasmidencoded sulfamethoxazole-trimethoprim resistance genes are prevalent in Malawi and the subsequent acquisition of these genes by this isolate could limit the treatment options available.

Sm-MW08 exhibited resistance to all $\beta$-lactam antibiotics and to $\beta$-lactamase inhibitors (Figure 1); this was consistent with the co-presence of chromosomally-encoded L1 metallo$\beta$-lactamase and L2 serine- $\beta$-lactamase. We consider that the resistance to other antibiotics, such as aminoglycosides, fluoroquinolones, and fosfomycin, was presumably due to low membrane permeability and/or derepression of chromosomally encoded multidrug resistance efflux pumps, as previously reported ${ }^{5}$. Notably, Sm-MW08 was devoid of a plasmid, further suggesting that the MDR-phenotype in SmMW08 was innate. Remarkably, S. maltophilia has been isolated from various hospital devices and can survive in various nutrient-poor environments (such as bottled water and handwashing soap) $)^{3}$, thus making $S$. maltophilia a significant issue of concern in clinical settings. Previous research found that antimicrobial susceptibility profiles vary between clinical and environmental isolates of $S$. maltophilia ${ }^{3}$, thus highlighting the need for susceptibility testing to guide the appropriate selection of antibiotics. S. maltophilia is commonly associated with immuno-incompetence; however, no clinical data were available for the patient at the time this study was carried out. Nevertheless, the recovery of this clinical isolate at $\mathrm{KCH}$ is of significant clinical importance.

\section{Conclusion}

We have described an MDR S. maltophilia isolate from Malawi. The isolate was resistant to almost all antibiotics tested. We therefore, recommend the implementation and practice of effective infection prevention measures to curtail the spread of this isolate.

\section{Competing interests}

The authors declare that they have no conflicts of interest.

\section{Acknowledgements}

We are grateful for the advice and comments made by Dr. Yo Sugawara and Dr. Yukihiro Akeda. We would also like to thank Akiko Ueda, Yumi Sasaki, and Kuzuhiro Maeda for their technical assistance with regards to species identification and antimicrobial susceptibility testing. The study was performed as part of the National Antimicrobial Surveillance Program by the Malawi National Microbiology Reference Laboratory.

\section{References}

1. Kim EJ, Kim YC, Ahn JY, Jeong JS, Ku NS, Choi JY, et al. Risk factors for mortality in patients with Stenotrophomonas maltophilia bacteremia and clinical impact of quinolone-resistant strains. BMC Infect Dis. 2019;19(1):1-8. doi:10.1186/s12879-019-4394-4

2. World Health Organization. Drug resistance [Internet]. 2019. Available from: https://www.who.int/drugresistance/AMR_Importance/en/

3. Adegoke AA, Stenström TA, Okoh AI. Stenotrophomonas maltophilia as an emerging ubiquitous pathogen: looking beyond contemporary antibiotic therapy. Clin Microbiol Rev. 2017;8(1):2276. doi:10.3389/ fmicb.2017.02276

4. Rabenandrasana MAN, Andrianoelina V, Bonneault M, Herindrainy P, Garin B, Breurec S, et al. Clonal transmission and new mechanism of resistance to trimethoprim-sulfamethoxazole in Stenotrophomonas maltophilia strains isolated in a neonatology unit at Antananarivo, Madagascar, deciphered by whole genome sequence analysis. bioRxiv. 2019;10(4):696765. doi:10.1101/696765

5. Bostanghadiri N, Ghalavand Z, Fallah F, Yadegar A, Ardebili A, Tarashi S, et al. Characterization of phenotypic and genotypic diversity of Stenotrophomonas maltophilia strains isolated from selected hospitals in Iran. Front Microbiol. 2019;10(1):1191. doi:10.3389/ fmicb.2019.01191 